\title{
CUANTIFICACIÓN DE PLOMO EN CERVEZA ARTESANAL MEDIANTE VOLTAMETRÍA DE REDISOLUCIÓN ANÓDICA CON PULSO DIFERENCIAL SOBRE ELECTRODO DE DIAMANTE DOPADO CON BORO
}

\section{LEAD CUANTIFICATION ON CRAFT BEER BY DIFFERENTIAL PULSE ANODIC STRIPPING VOLTAMMETRY ON BORON-DOPED DIAMOND ELECTRODE}

\author{
Ricardo Borja ${ }^{1}$, Lenys Fernandez ${ }^{1, *} \&$ Patricio Espinoza-Montero ${ }^{1, *}$
}

Recibido: 30 de abril 2020 / Aceptado: 30 de junio 2020

Publicado en línea: 17 de julio 2020

DOI: 10.26807/ia.v8i2.166

Palabras Clave: Cerveza Artesanal; DPASV; FAAS; Plomo

Key Words: Craft Beer, DPASV; FAAS; Lead

\section{RESUMEN}

Se reporta el estudio del contenido de $\mathrm{Pb}(\mathrm{II})$ en seis marcas de cervezas artesanales de la ciudad de Quito-Ecuador, utilizando Voltametría de Redisolución

1 Pontificia Universidad Católica del Ecuador, Facultad de Ciencias Exactas y Naturales, Escuela de Ciencias Químicas, Quito, Ecuador (ricardo-1395@hotmail.com; *correspondencia: pespinoza646@puce. edu.ec; Imfernandez@puce.edu.ec) 
Anódica con Pulso Diferencial (DPASV, por sus siglas en inglés), diamante dopado con boro (BDD, por sus siglas en inglés) como electrodo de trabajo y buffer de acetato como solución electrolítica. La caracterización del BDD se llevó a cabo mediante Microscopía Electrónica de Barrido (SEM, por sus siglas en inglés) y Voltamperometría Cíclica (VC). Los resultados por SEM mostraron que la superficie de electrodos de BDD es rugosa y granular, la cual presentó una baja corriente capacitiva y una respuesta electroquímica quasi-reversible frente al par redox Ferri/Ferrocianuro de potasio. El método se optimizó en un rango lineal entre 0,3-1,3 $\mathrm{mg} \mathrm{L}^{-1}$, se obtuvo repetibilidad con un RSD del 4,56\%, reproducibilidad del 9,19 \% y límite de detección de 0,020 $\mathrm{mg} \mathrm{L}^{-1}$. Las cervezas analizadas fueron etiquetadas como A, B, C, D, E y F, preservando así la identidad de las marcas que participaron en el estudio. Las muestras de cerveza $B$, C, D y E cumplen con la normativa NTE INEN 2262 para $\mathrm{Pb}$ (II) con una concentración por debajo de su límite máximo permitido de $0,1 \mathrm{mg} \mathrm{L}^{-1}$; mientras las muestras A y $\mathrm{F}$ exceden del límite máximo permisible. El método se validó mediante la comparación de los resultados obtenido por DPASV y Espectroscopia de Absorción Atómica por Llama (FAAS, por sus siglas en inglés), donde pruebas $t$-student indican que no hay diferencia significativa para la determinación del $\mathrm{Pb}$ (II) entre los métodos. Se concluye que la técnica de DPASV es una alternativa efectiva para la determinación de $\mathrm{Pb}$ (II) en cervezas artesanales.

\section{ABSTRACT}

Lead content was determined in six brands of craft beers from Quito-Ecuador, using Differential Pulse Anodic Stripping Voltammetry (DPASV), boron-doped diamond (BDD) as working electrode and acetate buffer as electrolytic solution. BDD was characterized using Scanning Electron Microscopy (SEM) and Cyclic Voltammetry (CV). The SEM results showed that its surface is rough and granular, where it presented a low capacitive current and a quasi-reversible electrochemical response against the Potassium Ferri/Ferrocyanide redox pair. The method was optimized with defined electrochemical signals from the analyte, a linear range between $0.3-1.3 \mathrm{mg} \mathrm{L}^{-1}$, repeatability with RSD of $4.56 \%$, reproducibi- 
lity with RSD of $9.19 \%$ and a detection limit of $0.020 \mathrm{mg} \mathrm{L}^{-1}$. The analyzed beers were labeled A, B, C, D, E and F, to preserve the brand identity for this study. Samples B, C, D and E comply with the NTE INEN 2262 norm for lead, whose value is below its maximum allowed limit of $0.1 \mathrm{mg} \mathrm{L}^{-1}$; while samples $A$ and $F$ exceed that limit. The method was validated by comparing results with respect to Flame Atomic Absorption Spectrophotometry (FAAS), where the tstudent test indicates that there was no significant difference for both methods. It is concluded that the DPASV technique is an effective alternative for the determination of lead in craft beers.

\section{INTRODUCCIÓN}

La cerveza artesanal es una de las bebidas más consumidas en el mundo; preparada de manera casera, a partir de la cebada, lúpulo, agua y levadura. Se ha incrementado su producción en los últimos años, debido a que es posible obtenerla manualmente, es decir, sin procesamiento industrial. Sin embargo, su buena calidad puede verse afectada por la presencia de metales pesados en su contenido (Passaghe et al., 2015; Corro y Vásquez, 2010).

Particularmente, el plomo, en ese contenido, puede provenir de la contaminación de la materia prima de esta bebida. El plomo es un metal pesado que puede actuar como neuro- toxina, produciendo graves e irreversibles daños a la salud humana. Sus signos y síntomas de contaminación en seres humanos se reflejan en problemas de comportamiento, anemia y daño renal que puede ocasionar la muerte (Fennema et al., 2010).

Se han reportado varios métodos de análisis para la determinación de estos metales pesados en cervezas, tales como: Espectrofotometría de Absorción Atómica (AAS) (Bellido et al., 2000), Espectrofotometría de Emisión Atómica (AES), Espectrofotometría de Emisión Óptica - acoplado al Plasma Inducido (ICP-OES) (Matsushigue y de Olivera, 1993), Espectrofotometría de Absorción Atómica con 
Horno de Llama de Termospray (TSFF-AAS) y Cromatografía lónica (IC) (Michalski et al., 2009). Sin embargo, estas técnicas son muy costosas debido a la instrumentación compleja que las constituyen y la poca portabilidad que pueden ofrecer. Como una técnica de análisis alternativa, se utiliza de Voltametría de Redisolución Anódica (ASV, por sus siglas en inglés), la cual consiste en la aplicación de un potencial para medir la señal de corriente del analito, acumulado previamente sobre la superficie del electrodo de trabajo mediante un paso de pre-concentración potenciostático.

En el presente estudio, para obtener la señal que corresponde al analito, se utilizó voltametría de pulso diferencial (DPV, por sus siglas en inglés), en la cual se aplican pulsos de potencial que permiten medir la corriente farádica, sin interferencia de la corriente capacitiva de acumulación de carga en la superficie del electrodo (Skoog et al., 2014).

Anteriormente, para la determinación electroanalítica de metales pesados, se utilizaba electrodos de trabajo de gota de mercurio (HMDE); debido a que ofrecían la redisolución rápida del metal a partir de su superficie, amplia ventana de potencial de reducción (potenciales negativos) y bajas corrientes de fondo (corriente capacitiva) para la determinación de los analitos a escala de traza (Zapata et al., 2016). Sin embargo, su uso ya no es recomendable en estos días, debido a que el mercurio es muy tóxico. Debido a la problemática con el mercurio, se han planteado electrodos de trabajo alternativos, tales como: bismuto, materiales carbonáceos como grafito, carbón vítreo, pastas de carbono, carbono impreso y BDD. Este último es un material que contiene carbono $\mathrm{sp}^{2}$, generado como contaminante del carbono $\mathrm{sp}^{3}$ del diamante al momento de doparlo con boro. El boro se inyecta durante la síntesis del diamante mediante deposición con vapor químico (CVD, por sus siglas en inglés), este se establece en la estructura cristalina del diamante, tomando el nombre BDD. Al contrario que el diamante el BDD es conductor y apropiado para ser utilizado como electrodo (Liu et al., 2016; Xu et al., 1997).

En este trabajo se reporta la cuantificación de $\mathrm{Pb}$ (II) en cerveza artesanal 
mediante DPASV utilizando un electrodo de trabajo de BDD. Hasta el momento no existe reportes de este tipo de estudios.

\section{MATERIALES Y MÉTODOS}

\section{Instrumentación}

Microscopio de barrido de electrones (SEM Phenom Pure) con detector de electrones secundarios; plancha de calefacción MTOP MS100 (HP 100), baño de ultrasonido Branson 3800; Potenciostato PGSTAT204 de Autolab Metrohm, celda electroquímica en forma de corazón, BDD como electrodo de trabajo, $\mathrm{Ag} / \mathrm{AgCl}$ como electrodo de referencia y mina de grafito como contraelectrodo; Espectrofotómetro de absorción atómica Perkin Elmer AAnalyst 400; y pH - metro Melter Toledo.

\section{Reactivos}

Cloruro de potasio, 99,99 \% (Fischer; Grado Analítico); ácido clorhídrico, 37 \% (Fischer; Grado Analítico); ácido nítrico, 69,2\% (Fischer; Grado Analítico); ácido acético glacial, 99,99\% (Merck, Grado Analítico); acetato de sodio (Loba Chemie, Grado Reactivo); y ferrocianuro de potasio, 99,99\% (Merck, Grado Analítico).

\section{Toma de muestras}

Las muestras de cerveza artesanal se obtuvieron mediante un muestreo aleatorio, de bares y locales de consumo, de tres diferentes sectores de Quito-Ecuador: La "Y", La Foch y La Pradera. Las diferentes marcas de cervezas analizadas fueron etiquetadas como: A, B, C, D, E y F, preservando así la identidad de las marcas que participaron en el estudio.

\section{Caracterización superficial del elec- trodo de trabajo}

La superficie del electrodo de diamante dopado con boro (BDD) fue caracterizado mediante SEM, aplicando un voltaje de resolución de 15 kV para la obtención de la imagen de su topología.

\section{Caracterización electroquímica del electrodo BDD mediante voltampe- rometría cíclica}

La caracterización del electrodo se 
realizó mediante voltamperometría cíclica, se empleó como electrolito de soporte de $\mathrm{KCl} \mathrm{0,1} \mathrm{mol} \mathrm{L}^{-1}$, un electrodo de referencia de $\mathrm{Ag} / \mathrm{AgCl}$ (con $\mathrm{KCl} 3 \mathrm{~mol} \mathrm{~L}^{-1}$ ) y un contraelectrodo de grafito.

La ventana de potencial de trabajo del electrodo fue determinada por medio de un barrido de potencial cíclico, a una velocidad de $0,1 \mathrm{~V} \mathrm{~s}^{-1}$.

Se determinó la capacitancia de doble capa con el electrolito soporte en un rango de velocidad de barrido entre $0,025-0,125 \mathrm{~V} \mathrm{~s}^{-1}$. Además, se estudió el comportamiento de transferencia de electrones del par redox Ferri/Ferro a través de la constante de velocidad de intercambio $\left(k^{\circ}\right)$, calculado a partir de la ecuación (ec. 1) y el método de Nicholson (Nicholson, 1965); para ello se utilizó una solución de ferrocianuro de potasio 4 mmol L-1 en $\mathrm{KCl} 0,1 \mathrm{~mol} \mathrm{~L}^{-1}$ en un rango de velocidad de barrido de 0,01 a $0,1 \mathrm{~V} \mathrm{~s}^{-1}$.

$$
k^{0}=\frac{\psi\left(\pi D_{0} F v\right)^{1 / 2}}{(R T)^{1 / 2}}
$$

Donde, $\boldsymbol{k}^{\boldsymbol{o}}$ es la constante de velocidad de intercambio de electrones, $\boldsymbol{\Psi}$ es la función de la constante hetero- génea de velocidad del electrón, $\boldsymbol{D}_{\boldsymbol{0}}$ es la constante de la especie química que realiza el proceso redox, $\boldsymbol{v}$ es la velocidad de barrido, $\boldsymbol{F}$ es constante de Faraday, $\boldsymbol{R}$ es la constante universal de los gases y $\boldsymbol{T}$ es la temperatura $\left(25^{\circ} \mathrm{C}\right)$.

\section{Validación de la determinación de $\mathrm{Pb}$ (II) por DPASV}

Se construyó una curva de calibración de $\mathrm{Pb}$ (II), en un rango de concentraciones de 0,25 a 1,25 $\mathrm{mg} \mathrm{L}^{-1}$. Se determinó el rango lineal, límite de detección y cuantificación, repetibilidad, reproducibilidad y exactitud.

\section{Tratamiento de las muestras reales}

Se realizó una digestión ácida abierta, para eliminar la posible presencia de especies interferentes en las muestras de cerveza artesanal. Para esto, se tomaron alícuotas de $25 \mathrm{~mL}$ de la muestra, se desgasificaron mediante baño de ultrasonido, se mezclaron con $\mathrm{HNO}_{3}$ al $67 \%$, se calentaron lentamente en una plancha calefactora, se aforaron a $50 \mathrm{~mL}$ con buffer de acetato $0,1 \mathrm{~mol} \mathrm{~L}^{-1}$, a pH de 4,5; y se diluyeron en el buffer de acetato con un factor de dilución de 1:50. 
Todas las muestras fueron fortificadas con soluciones estándar de $\mathrm{Pb}, \mathrm{a}$ concentración conocida, de manera independiente y dentro del rango lineal establecido para este estudio (Nascentes et al., 2005).

\section{Determinación electroanalítica de $\mathrm{Pb}$ (II) mediante DPASV y FAAS}

Las muestras fueron analizadas por DPASV y por FAAS. Los resultados obtenidos fueron comparados mediante pruebas de $t$-student para varianzas desiguales.
La detección de $\mathrm{Pb}$ (II) por DPASV se realizó a potencial de pulso y amplitud de 0,01 y 0,05 $\mathrm{V}$ respectivamente, y a tiempo de modulación e intervalo de 0,05 y 0,5 s respectivamente. En el caso de la detección por FAAS se utilizó una lámpara de cátodo hueco multielemental, a una longitud de onda de $217 \mathrm{~nm}$, Slit de 0,5 a $2 \mathrm{~mm}$ y con flujo de aire comprimido-acetileno en proporción 10:2

\section{RESULTADOS}

Caracterización superficial del electrodo

En la Figura 1 se observa la imagen del electrodo BDD, obtenida por SEM.

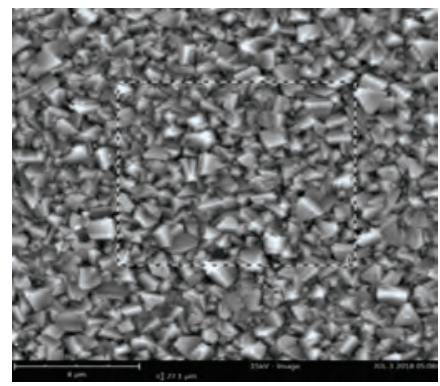

Figura 1. Imagen SEM de un electrodo de BDD

\section{Caracterización electroquímica del electrodo}

En la Figura 2, se muestra el comportamiento voltamperométrico del BDD en el medio electrolítico, con una ventana de potencial de $-1,2$ a $1,2 \mathrm{~V}$ 


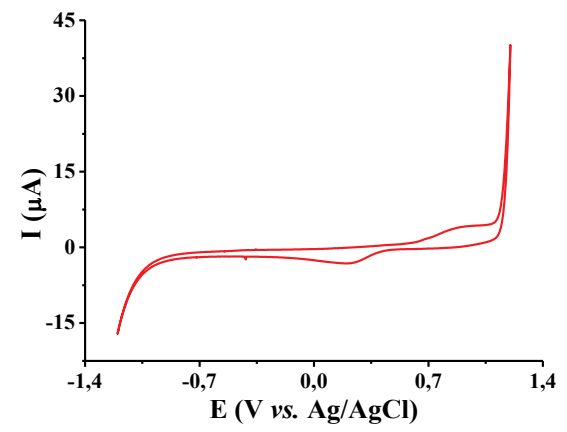

Figura 2. Voltamperograma cíclico del $\mathrm{BDD}$ en $\mathrm{KCl} 0,1 \mathrm{~mol} \mathrm{~L}^{-1}$, a velocidad de barrido de $100 \mathrm{mV} \mathrm{s}^{-1}$
La Figura 3 muestra una serie de voltamperogramas cíclicos sobre el electrodo de BDD, en $\mathrm{KCl} 0,1 \mathrm{~mol} \mathrm{~L}^{-1}$, a diferentes velocidades de barrido $y$ en su inserto se observa la curva de densidad de corriente $v s$. velocidad de barrido de los voltamperogramas anteriores.

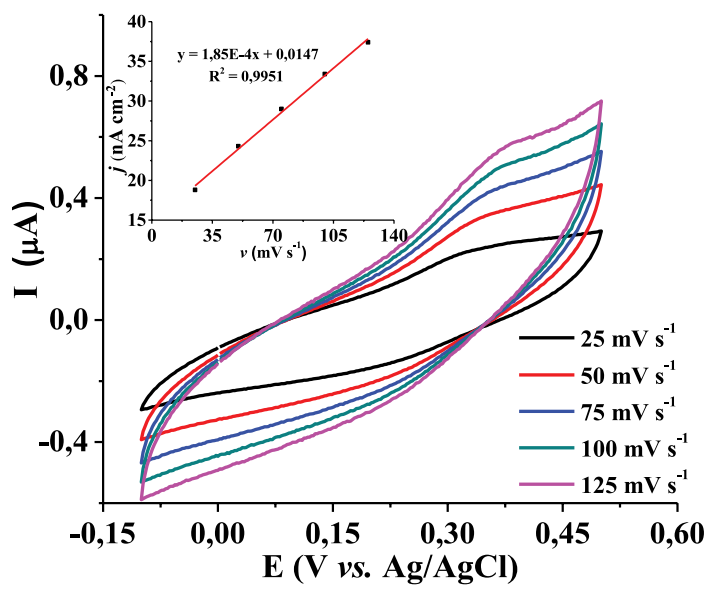

Figura 3. Voltamperogramas cíclicos del BDD en $\mathrm{KCl} \mathrm{mol} \mathrm{L-1} \mathrm{pH}=1$, a distintas velocidades de barrido. Inserto, curva $j$ vs. $v$, tomada a $0,16 \mathrm{~V}$

La Figura 4, muestra los voltamperogramas cíclicos del electrodo BDD en solución de $\mathrm{Fe}(\mathrm{CN})_{6}^{3-4-} 4 \mathrm{mmol} \mathrm{L}^{-1}$ en $\mathrm{KCl} 0,1 \mathrm{~mol} \mathrm{~L}^{-1}$. A partir de esta fi- gura se construyó la curva Intensidad de corriente vs. la raíz cuadrada de la velocidad de barrido (inserto). 


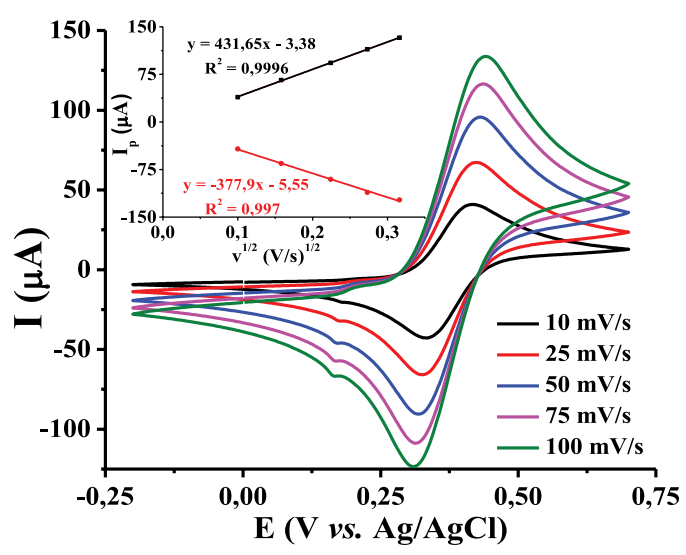

Figura 4. Comportamiento del $\mathrm{K}_{3} \mathrm{Fe}(\mathrm{CN})_{6} 4 \mathrm{mmol} \mathrm{L}^{-1}$ en $\mathrm{BDD}, \mathrm{KCl} \mathrm{mol} \mathrm{L-1}, \mathrm{pH}=1$, a distintas velocidades de barrido. Inserto, curvas de $I_{p} v s . v^{1 / 2}$

Determinación electroanalítica de $\mathrm{Pb}$ (II) sobre BDD mediante DPASV

La Figura 5, muestra la señal de redisolución del Pb(II) por DPASV y en el inserto se detalla la curva de calibración respectiva. La Figura 6 indica el estudio del efecto matriz, a partir de curvas de calibración construidas con y sin fortificación.

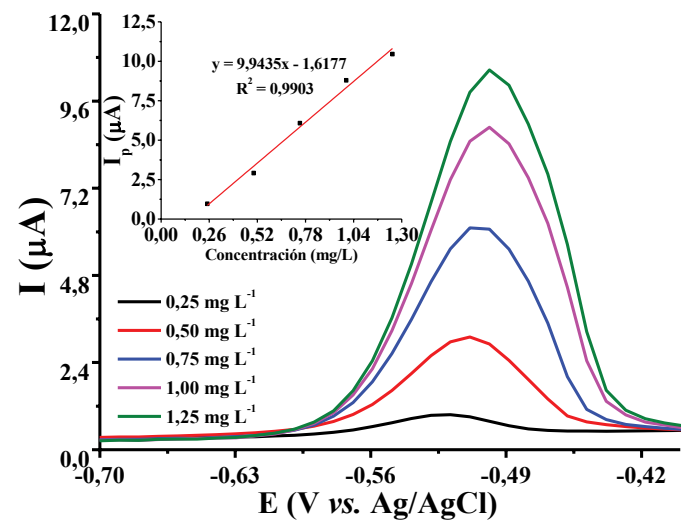

Figura 5. Voltamperograma de pulso diferencial de $\mathrm{Pb}$ (II) en Buffer ácido acético/acetato de sodio $0,1 \mathrm{M} \mathrm{a} \mathrm{pH}=4,5$. Inserto, curva de calibración 


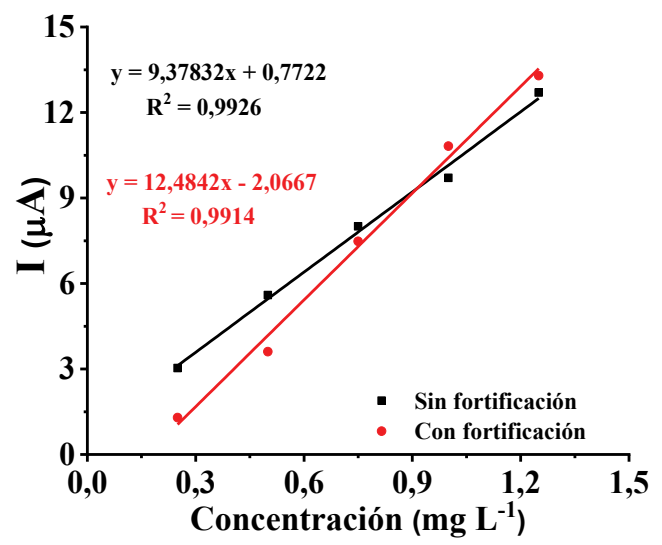

Figura 6. Curvas de calibración para $\mathrm{Pb}(\mathrm{II})$, sin y con fortificación de la muestra, por DPASV en BDD

La información de la Tabla 1, resume el comportamiento redox del $\mathrm{Fe}(\mathrm{CN})_{6}^{3-14-}$ sobre el electrodo BDD.

Las figuras de mérito obtenidas en la evaluación del método de detección de $\mathrm{Pb}$ (II) por DPASV se muestran en la Tabla 2. Mientras que en la Tabla 3 , se presenta el contenido de $\mathrm{Pb}$ (II) analizado por DPASV y FAAS para cada muestra analizada, con el límite máximo permisible según la norma NTE INEN:2262 y su cumplimiento.

Tabla 1. Resumen de los datos de la Voltamperometría Cíclica del $\mathrm{K}_{3} \mathrm{Fe}(\mathrm{CN})_{6}$ en el electrodo de BDD

\begin{tabular}{cccccc}
\hline $\begin{array}{c}\mathbf{V} \\
\left(\mathrm{mV} \mathrm{s}^{-1}\right)\end{array}$ & $\begin{array}{c}\mathbf{E}_{\mathrm{p}^{1} / 2} \\
(\mathrm{mV})\end{array}$ & $\begin{array}{c}\Delta \mathrm{E}_{\mathrm{p}} \\
(\mathrm{mV})\end{array}$ & $\begin{array}{c}\mathrm{E}_{\mathrm{p}, \mathrm{ox}} \\
(\mathrm{mV})\end{array}$ & $\mathrm{I}_{\mathrm{p}, \mathrm{ox}} / \mathrm{I}_{\mathrm{p}, \mathrm{red}}$ & $\begin{array}{c}\mathbf{k}^{\circ} \\
\left(\mathrm{cm} \mathrm{s}^{-1}\right)\end{array}$ \\
\hline 10 & 362,5 & 107 & 416 & 0,92 & $1,52 \times 10^{-3}$ \\
25 & 376,0 & 102 & 425 & 0,99 & $2,41 \times 10^{-3}$ \\
50 & 373,5 & 115 & 427 & 0,97 & $3,41 \times 10^{-3}$ \\
75 & 372,5 & 123 & 436 & 1,03 & $2,92 \times 10^{-3}$ \\
100 & 375,0 & 139 & 395 & 1,08 & $2,41 \times 10^{-3}$ \\
\hline
\end{tabular}


Tabla 2. Parámetros analíticos para el Pb(II), medidos por DPASV $(n=3)$

\begin{tabular}{lc}
\hline Parámetro & $\mathbf{P b}(\mathrm{II})$ \\
\hline Rango lineal (mg L-1) & $0,25-1,25$ \\
Coeficiente de correlación ( ${ }^{2}$ ) & 0,9903 \\
Límite de Detección (este trabajo) (mg L-1) & 0,020 \\
Límite de Detección (FAAS, Nascentes et al., 2005) (mg L-1) & 0,046 \\
Límite de Cuantificación (este trabajo) (mg L ${ }^{-1}$ ) & 0,066 \\
Límite de Cuantificación (FAAS, este trabajo) & 0,153 \\
Repetibilidad concentraciones bajas (\%RSD) (mg L-1) & 10,53 \\
Repetibilidad concentraciones altas (\%RSD) & 4,56 \\
Reproducibilidad (\%RSD) & 9,19 \\
\hline
\end{tabular}

Tabla 3. Contenido de $\mathrm{Pb}$ (II) obtenido en diferentes marcas de cerveza artesanal

\begin{tabular}{ccccccc}
\hline Marca & $\begin{array}{c}\text { DPASV } \\
\left(\mathrm{mg} \mathrm{L}^{-1}\right)\end{array}$ & \% RSD & $\begin{array}{c}\text { FAAS } \\
\left(\mathrm{mg} \mathrm{L}^{-1}\right)\end{array}$ & \% RSD & $\begin{array}{c}\text { Lím. máx. } \\
\text { Permitido } \\
\left(\mathrm{mg} \mathrm{L}^{-1}\right)\end{array}$ & $\begin{array}{c}\text { Cumple con la } \\
\text { norma INEN } \\
\mathbf{2 2 6 2}\left(^{*}\right)\end{array}$ \\
\hline A & 0,2740 & 4,82 & 0,0672 & 5,36 & 0,1000 & No \\
B & 0,0663 & 5,86 & 0,0785 & 3,89 & 0,1000 & Sí \\
C & 0,0843 & 4,18 & 0,0635 & 5,06 & 0,1000 & Sí \\
D & 0,0959 & 4,06 & 0,0955 & 5,43 & 0,1000 & Sí \\
E & 0,0448 & 5,6 & 0,0642 & 4,67 & 0,1000 & Sí \\
F & 0,1615 & 6,12 & 0,0650 & 5,69 & 0,1000 & No \\
\hline
\end{tabular}

*El criterio expuesto se realizó al comparar con los resultados obtenidos mediante la técnica de DPASV

\section{DISCUSIÓN}

La morfología del electrodo BDD no presenta fisuras en su superficie, lo que sugiere que es apto para las determinaciones electroquímicas. El co- lor obscuro de los cristales de diamante se asocia al dopado con boro durante su construcción y a la presencia de carbono $\mathrm{sp}^{2}$, Figura 1 
(Macpherson, 2015). La Figura 2 presenta una ventana de potencial de trabajo de 1,20 a -1,20 V, la cual es más estrecha que la reportada por Show et al. (Show et al., 2003), y por Xu et al. para el BDD de alta calidad (Xu et al., 1997). Además, se observa la señal el potencial de evolución de oxígeno a 1,2 V y la señal de desorción de oxígeno de la superficie del electrodo, a 0,3 V (Sartori et al., 2018). En la Figura 3, la densidad de corriente incrementó linealmente con la velocidad de barrido con un factor de correlación de 0,9951 (inserto de la figura), se obtuvo un valor de capacitancia del electrodo es de $0,0002 \mu \mathrm{F} \mathrm{cm}^{-2}$, siendo este menor al valor reportado por Dejmkova et al., de 15,2 $\mu \mathrm{F} \mathrm{cm}^{-2}$; esta diferencia se puede asociar a la resistencia capacitiva y a la baja concentración de C$\mathrm{sp}^{2}$ que podría presentar el electrodo de BDD utilizado en el análisis (Dejmkova et al., 2009; Liu et al., 2016; Kim et al., 2013). Los picos, anódico y catódico (Figura 4), son bien definidos y el potencial de oxidación y reducción varían en aproximadamente $0,1 \mathrm{~V}$ al incrementar la velocidad de barrido, lo que indica que no hay complicaciones cinéticas en el proceso redox del par ferri-ferro
$\left(\mathrm{Fe}(\mathrm{CN})_{6}^{3-/ 4-}\right)$ sobre la interfase del BDD (Yu et al., 2012). La variación lineal de la corriente, tanto anódica como catódica, con respecto a la raíz cuadrada de la velocidad de barrido (inserto de la Figura 4), indica que la reacción redox sobre la superficie del BDD, es química y electroquímicamente reversible, y está controlada por la difusión linear semi-infinita del $\mathrm{Fe}(\mathrm{CN})_{6}{ }^{3-/ 4-}$ (Show et al., 2002). Debido a que el comportamiento redox del $\mathrm{Fe}(\mathrm{CN})_{6}^{3-/ 4-}$ está gobernado por un proceso de transferencia de electrones de esfera interna, la fracción expuesta de carbono $\mathrm{C}-\mathrm{sp}^{2}$ sobre el BDD lo hace un electrodo muy sensible a la limpieza, no obstante, el comportamiento obtenido, sugiere que el BDD utilizado respondió efectivamente al proceso electroquímico sometido (Show et al., 2002). El aumentó ligeramente con el incremento de la velocidad de barrido (Tabla 1), indicando un control adecuado de la caída óhmica (resistencia del electrolito) y la ausencia de complicaciones cinéticas ocasionadas por reacciones químicas que pudiesen ocurrir en el medio electrolítico, la presencia de oxígeno o modificaciones superficiales del electrodo a niveles microscópicos (García - Jareño et al., 1998; 
Swain, 2004). Los valores de $\mathrm{k}^{\circ}$ varían ligeramente al incrementar la velocidad de barrido, lo cual es característico de electrodos rugosos como el BDD (Fischer et al., 2004; Alehashem et al., 1995). Estos valores están dentro del rango de $1 \times 10^{-6} \mathrm{a}$ $1 \times 10^{-2} \mathrm{~cm} \mathrm{~s}^{-1}$, particular del BDD de alta calidad frente al sistema redox $\mathrm{Fe}(\mathrm{CN})_{6}^{3-14-}$ (Granger et al., 2000). La Figura 5 muestra la señal de redisolución del plomo por PDV, donde se observa una señal simétrica que aumenta con la concentración de plomo en solución, con un factor de correlación de 0,9903 y una sensibilidad de $9,9 \mathrm{~mA} / \mathrm{mgL}^{-1}$ (inserto de la figura), lo que demuestra un comportamiento lineal favorable de la señal medida con la concentración de $\mathrm{Pb}$ en solución. En el estudio de efecto matriz, Figura 6, se encontró que hay poca influencia de la matriz, donde se encuentra la cerveza, sobre las medidas, ya que no hay diferencia significativa en la sensibilidad de la curva de calibración cuando la muestra se encuentra en presencia de los estándares fortificados a cuando se está en ausencia de ellos (Pretty et al., 1990; Brooksh y Kovalski, 1994). De acuerdo con estos resultados, se utilizó la ecuación de la curva con fortificación para la cuantificación de las muestras. El LD y LC obtenido por DPASV, son más bajos con respecto a la técnica de FAAS, Tabla 2 (Nascentes et al., 2005). En los estudios de repetibilidad y reproducibilidad del método, para un análisis por triplicado, se encontró que la señal es repetible (RSD de 4,56 \%) sólo a concentraciones altas de la curva de calibración (curva optimizada) y reproducible para la detección en cerveza, a concentración de 0,875 mg L-1, con un RSD de 9,19\%. La Tabla 3, muestra que cervezas etiquetadas como B, C, D y E cumplen con la normativa NTE INEN 2262, ya que no exceden el límite máximo permisible por esta norma, sugiriendo que el tratamiento de la muestra fue efectivo para la eliminación de la matriz orgánica (Matusiewicz y Kopras, 1997). Por otro lado, las cervezas A y F exceden del límite máximo permitido de $\mathrm{Pb}$ (II) para esta normativa. Sin embargo, debemos destacar que el valor permisible para $\mathrm{Pb}(\mathrm{II})$ en la norma NTE INEN 2262 es menor con respecto a la normativa mundial del Office International de la Vigne et du Vin (OIV, 2014; Ghanjaoui et al., 2011), el cual se establece de 0,2 mg $\mathrm{L}^{-1}$. De acuerdo a la prueba estadís- 
tica $t$-student, no hay diferencia significativa en la comparación de los resultados obtenidos por DPASV con respecto a lo obtenido por FAAS, en la determinación de contenido del $\mathrm{Pb}$ (II) en cerveza artesanal.

\section{CONCLUSIÓN}

La caracterización superficial y electroquímica del electrodo de BDD sugieren que éste es un electrodo apto para su empleo en la determinación de $\mathrm{Pb}$ en muestras de cervezas artesanales. La DPASV sobre electrodos de BDD, es una técnica alternativa, innovadora y apropiada para la determinación de $\mathrm{Pb}$ (II) en muestras de cerveza artesanal de la ciudad de Quito, Ecuador. Los resultados obtenidos con el método optimizado por
DPASV no mostraron diferencia significativa con resultados obtenidos por FAAS. Las muestras de cerveza B, C, D y E cumplen con la normativa NTE INEN 2262 para $\mathrm{Pb}(\mathrm{II})$ al tener un valor por debajo del límite máximo permitido por esta norma. Mientras, las muestras A y F no cumplen con la normativa ecuatoriana, pero si con la normativa internacional.

\section{LISTA DE REFERENCIAS}

Bellido, D., Moreno, J. y Hernández, M. (2000) Differentiation and classification of beers with flame atomic spectrometry and molecular absorption spectromeetry and sample preparation assisted by microwaves. Spectrochimica Acta Part B 55, 855 - 864.

Booksh, K. S., y Kowalski, B. R. (1994). Theory of analytical chemistry. Analytical Chemistry, 66(15), 782A-791A. doi: 10.1021/ac00087a001

Corro, M. y Vásquez, V. (2010) Control automático con lógica difusa de la producción de cerveza artesanal en las etapas de maceración y cocción. Scientia Agropecuaria (1) $125-137$. 
Dejmkova, H., Scampicchio, M., Zima, J., Barek, J., y Mannino, S. (2009). Determination of Total Phenols in Foods by Boron Doped Diamond Electrode. Electroanalysis, 21(9), 1014-1018.doi:10.1002/elan.200804508

El Tall, O., Jaffrezic-Renault, N., Sigaud, M., y Vittori, O. (2007). Anodic Stripping Voltammetry of Heavy Metals at Nanocrystalline Boron-Doped Diamond Electrode. Electroanalysis, 19(11), 1152-1159. doi:10.1002/elan.200603834

Garcia-Jareno, J. J., Benito, D., Navarro-Laboulais, J. y Vicente, F. (1998). Electrochemical Behavior of Electrodeposited Prussian Blue Films on ITO Electrode: An Attractive Laboratory Experience. Journal of Chemical Education, 75(7), 881. https://doi. org/10.1021/ed075p881

Ghanjaoui, M., Srji, M., Hor, M. Serdaoui, F. y El Rhazi, M. (2012). Fast procedure of lead determination in alcoholic beverages. J. Mater Enriron. Sci. 3 (1), 85-90.

Izah, S.; Inyang, I.; Angaye, T. y Okowa, T. (2016) A Review of Heavy Metal Concentration and Potential Health Implications of Beverages Consumed in Nigeria. Toxics. 5,1 .

Liu, D., Gou, L., Xu, J., Gao, K., y Kang, X. (2016). Investigations on etching resistance of undoped and boron doped polycrystalline diamond films by oxygen plasma etching. Vacuum, 128, 80-84. doi: 10.1016/j.vacuum.2016.03.012

Lukinac, J., Mastanjević, K., Mastanjevi॰, K., Nakov, G., y Jukiゅ, M. (2019). Computer Vision Method in Beer Quality Evaluation-A Review. Beverages, 5(2), 38. doi:10.3390/beverages5020038

Macpherson, J. V. (2015). A practical guide to using boron doped diamond in electrochemical research. Physical Chemistry Chemical Physics, 17(5), 29352949. doi:10.1039/c4cp04022h

Magnusson, B. y Oenemark, U. (Eds.) (2014) Eurachem Guide: The Fitness for Purpose of Analytical Methods - A laboratory Guide to Method Validation and Related Topics (2da Edición). 
Matsushigue, I. y de Oliveira, E. (1993) Determination of trace elements in Brazilian beers by ICP-AES. Food Chemistry 47, $205-207$.

Matusiewicz, H., \& Kopras, M. (1997). Methods for Improving the Sensitivity in Atom Trapping Flame Atomic Absorption Spectrometry: Analytical Scheme for the Direct Determination of Trace Elements in Beer. Journal of Analytical Atomic Spectrometry, 12(11), 1287-1291.doi:10.1039/a704407k

Michalski, R. (2009). Applications of lon Chromatography for the Determination of Inorganic Cations. Critical Reviews in Analytical Chemistry, 39(4), 230-250. doi:10.1080/10408340903032453

Nascentes, C., Kamogawa, M., Fernandes, K., Arruda, M., Nogueira, A. y Nobrega, J. (2005). Direct determination of $\mathrm{Cu}, \mathrm{Mn}, \mathrm{Pb}$ and $\mathrm{Zn}$ in beer by thermospray flame furnace atomic absorption spectrometry. Spectrochimica Acta part B. 60, 749 753.

NTE INEN 2262:2003, Bebidas alcohólicas, Cerveza, Requisitos.

Organisation Internationale de la Vigne et du Vin (OIV) (2014), Annexe C - Limites maximales acceptables de divers éléments contenus dans le vin, OIV-MA-C1-01, Volume 2, Paris, France

Passaghe, P., Bertoli, S., Tubaro, F., y Buiatti, S. (2015). Monitoring of some selected heavy metals throughout the brewing process of craft beers by inductively coupled plasma mass spectrometry. European Food Research and Technology, 241(2), 199215. doi:10.1007/s00217-015-2445-7

Pretty, J. R., Evans, E. H., Blubaugh, E. A., Shen, W.-L., Caruso, J. A., y Davidson, T. M. (1990). Minimisation of sample matrix effects and signal enhancement for trace analytes using anodic stripping voltammetry with detection by inductively coupled plasma atomic emission spectrometry and inductively coupled plasma mass spectrometry. Journal of Analytical Atomic Spectrometry, 5(6), 437. doi:10.1039/ ja9900500437 
Sartori, A., Orlando, S., Bellucci, A., Trucchi, D., Abrahami, S., Boehme, T. y Buijnsters, J. G. (2018). Laser-Induced Periodic Surface Structures (LIPSS) on Heavily BoronDoped Diamond for Electrode Applications. ACS Applied Materials \& Interfaces.doi:10.1021/acsami.8b15951

Show, Y., Witek, M. A., Sonthalia, P., y Swain, G. M. (2003). Characterization and electrochemical responsiveness of boron-doped nanocrystalline diamond thin-film electrodes. Chemistry of Materials, 15(4), 879-888. https://doi.org/10.1021/ $\mathrm{cm} 020927 \mathrm{t}$

Skoog, D. West, D., Holler, F. y Crouch, S. (2014). Fundamentals of Analytical Chemistry. 9na Edición. Cengage Learning. Estados Unidos

Swain, G. M. (2004). Chapter 4 Electroanalytical applications of diamond electrodes. Semiconductors and Semimetals, 121-148.doi:10.1016/s0080-8784(04)80016-4

Xu, J., Granger, M. C., Chen, Q., Strojek, J. W., Lister, T. E., y Swain, G. M. (1997). Peer Reviewed: Boron-Doped Diamond Thin-Film Electrodes. Analytical Chemistry, 69(19), 591A-597A. doi:10.1021/ac971791z

Yu, Z., Wang, J., Wei, Q., Meng, L., Hao, S., y Long, F. (2013). Preparation, characterization and electrochemical properties of boron-doped diamond films on $\mathrm{Nb}$ substrates. Transactions of Nonferrous Metals Society of China, 23(5), 1334-1341. doi:10.1016/s1003-6326 (13)62601-1

Zapata, E.; Gazcón, N. y Flores, L. (2016) A direct method for the determination of lead in beers by differential pulse polarography-anodic stripping voltammetry. J. Mater. Environ. Sci. 7 (12), 4467-4470 